\title{
Clinical Guideline Highlights for the Hospitalist: Therapeutic Monitoring of Vancomycin
}

\author{
Mark Murphy, DO 1,2*, Sonya Tang Girdwood, MD, PhD $2,3,4$, Marc H Scheetz, PharmD, MSc ${ }^{5,6,7}$
}

\begin{abstract}
'Division of Infectious Diseases, Cincinnati Children's Hospital Medical Center, Cincinnati, Ohio; ${ }^{2}$ Clinical Pharmacology, Cincinnati Children's Hospital Medical Center, Cincinnati, Ohio; ${ }^{3}$ Hospital Medicine, Cincinnati Children's Hospital Medical Center, Cincinnati, Ohio; ${ }^{4}$ Department of Pediatrics, University of Cincinnati College of Medicine, Cincinnati, Ohio; ${ }^{5}$ Pharmacometrics Center of Excellence, Department of Pharmacy Practice, College of Pharmacy, Midwestern University, Downers Grove, Illinois; ' ${ }^{6}$ epartment of Pharmacology, College of Graduate Studies, Midwestern University, Downers Grove, Illinois; ${ }^{7}$ Department of Pharmacy, Northwestern Medicine, Chicago, Illinois.
\end{abstract}

\begin{abstract}
GUIDELINE TITLE: Therapeutic monitoring of vancomycin for serious methicillin-resistant Staphylococcus aureus infections: A revised consensus guideline and review by the American Society of Health-System Pharmacists, the Infectious Diseases Society of America, the Pediatric Infectious Diseases Society, and the Society of Infectious Diseases Pharmacists
\end{abstract}

RELEASE DATE: Online: March 19, 2020

PRIOR VERSION: 2009
DEVELOPERS: American Society of Health-System Pharmacists (ASHP), the Infectious Diseases Society of America (IDSA), the Pediatric Infectious Diseases Society (PIDS), and the Society of Infectious Diseases Pharmacists (SIDP)

FUNDING SOURCE: ASHP, IDSA, PIDS, SIDP

TARGET POPULATION: Adults, children, and neonates treated for documented or presumed methicillin-resistant Staphylococcus aureus infection
V ancomycin, a glycopeptide antibiotic, has been used for decades, yet knowledge gaps remain regarding the most appropriate dosing approach to optimize therapeutic effect while avoiding adverse effects in all patient populations. A committee composed of members of the American Society of Health-System Pharmacists, the Infectious Diseases Society of America, the Pediatric Infectious Diseases Society, and the Society of Infectious Diseases Pharmacists reviewed data available since publication of the original 2009 vancomycin dosing guidelines to provide new recommendations regarding vancomycin dosing and serum concentrationmonitoring in the empiric treatment of presumed or confirmed methicillin-resistant Staphylococcus aureus (MRSA) infections. ${ }^{1}$

The new guidelines provide 25 recommendations encompassing the following topics: vancomycin dosing and monitoring in adult, pediatric, and neonate care; vancomycin minimum inhibitory concentration (MIC) susceptibility testing; continuous infusion vs intermittent infusion; loading doses; dosing in obesity; and dosing in patients on hemodialysis and continuous renal replacement therapy. Because hospitalists in pediatric and adult care frequently prescribe vancomycin for empiric and targeted treatment of serious infections, they have a vested interest in ensuring optimal vancomycin outcomes (ie, best efficacy with least toxicity) with use of therapeutic drug monitoring and personal-

*Corresponding Author: Mark Murphy, DO; Email: Mark.Murphy@cchmc.org; Telephone: 513-803-7421; Twitter: @_Mark_Murphy.

Published online first November 18, 2020

Received: April 14, 2020; Revised: June 18, 2020; Accepted: July 20, 2020

(C) 2020 Society of Hospital Medicine DOI 10.12788/jhm.3507 ized dosing of vancomycin. Thus, it is important for hospitalists to be aware of the updated guideline and pivotal changes regarding therapeutic drug monitoring. In this guideline review, we will focus on the major differences from the 2009 guideline, specifically regarding therapeutic monitoring in adults and children.

The guideline includes pharmacology language and terminology with which many clinicians may not be familiar. To better understand the rationale for the guideline changes, a few concepts will be reviewed. Overall, antibiotics are dosed based on preclinical studies to determine the needed drug exposure for optimal efficacy. $\beta$-Lactams, for example, are optimally dosed with longer drug exposure time above the MIC of the infectious organism. Alternatively, area under the concentration time curve (AUC) describes the efficacy and toxicity of many other antibiotics. Since AUC is derived from products of concentration ( $\mathrm{mg} / \mathrm{L}$ ) and time (hours), the units are often $\mathrm{mg} \times \mathrm{h} / \mathrm{L}$. For vancomycin, both drug exposure (ie, AUC) and organism susceptibility (ie, MIC) are incorporated to determine optimal drug exposure, with the ratio of AUC to MIC being the ideal marker. Therapeutic drug monitoring of vancomycin has classically been conducted with trough concentration monitoring, but with the updated guideline, there will be a transition to AUC monitoring that will affect patient care and experience.

\section{KEY RECOMMENDATIONS FOR HOSPITALISTS TREATING ADULTS}

The following is a summary of recommendations 1 to 6 :

- In adults, the optimal drug exposure for vancomycin should be an AUC to MIC ratio of 400 to 600 for MRSA, with the assumption of MIC to be $1 \mathrm{mg} / \mathrm{L}$ (evidence quality: A-II).

The preferred method to monitor AUC is with a clinical 
statistical software that uses two blood samples (1 to 2 hours after completion of infusion and at the end of a dosing interval [ie, trough]) (evidence quality: A-II).

- An alternative approach would be to use first-order pharmacokinetic equations at steady state with a peak and trough (evidence quality: A-II).

- These approaches replace the previously recommended trough-only monitoring. AUC-targeted exposure should generally be achieved within 48 hours; severity of infection does not justify higher AUC goals. Once the goal AUC is achieved, once-weekly monitoring is recommended for hemodynamically stable patients, but more frequent or daily monitoring is advised in patients at high risk of nephrotoxicity or who are hemodynamically unstable (evidence quality: B-II).

The currently accepted optimal drug exposure for vancomycin is an AUC to MIC ratio of 400 to 600 to maximize efficacy and minimize nephrotoxicity. ${ }^{2}$ Due to clinical inconvenience of performing AUC-based monitoring for vancomycin in the past, previous guidelines recommended using trough concentrations as a surrogate marker for an AUC to MIC ratio, with the goal trough being 15 to $20 \mathrm{mg} / \mathrm{L}$ for serious MRSA infections. ${ }^{3}$ However, trough values may not correlate well with AUC. For example, a trough of $15 \mathrm{mg} / \mathrm{L}$ may represent an AUC ranging from 400 to $1000 \mathrm{mg}$ $\times \mathrm{h} / \mathrm{L}$ over 24 hours. Without knowing an accurate AUC, there is risk for ineffective bactericidal activity with low AUCs or nephrotoxicity with high AUCs. Compared with trough-only monitoring, AUC-guided dosing is associated with decreased risk of acute kidney injury. ${ }^{4,5}$ Therefore, the recommendation to transition to two-sample collection with a peak and trough was included.

Software programs are now readily available to compute the AUC and work best with peak and trough values rather than a single trough value because computing with two concentrations will rely more on specific patient data than it does on previously published vancomycin models. Trough-only monitoring (and without the support of clinical software) may still be possible when the exposures needed are further from the toxic range. To this end, trough-only monitoring may be reasonable when infections are not MRSA and are less invasive (eg, cellulitis) since the guideline found insufficient evidence for AUC monitoring in these scenarios. While specific targets are not provided, a plethora of historical literature demonstrated low kidney injury rates when troughs were maintained between 5 to $10 \mathrm{mg} / \mathrm{L}$.

\section{KEY RECOMMENDATIONS FOR PEDIATRIC HOSPITALISTS}

The following is a summary of recommendations 18 to 20:

- In pediatric care, based on a target AUC to MIC ratio of 400 to 600 with the assumption of MIC to be $1 \mathrm{mg} / \mathrm{L}$, initial vancomycin dosage for MRSA is as follows (evidence quality: A-II) :

60 to $80 \mathrm{mg} / \mathrm{kg}$ per day, divided into four doses, each given 6 hours apart, for children 3 months and older but younger than 12 years

- 60 to $70 \mathrm{mg} / \mathrm{kg}$ per day, divided into four doses, each given 6 hours apart, for children 12 years and older

- As recommended in adults, use of a statistical software program to measure AUC is the optimal approach in pediatric care because it can account for age, weight, and renal function, which should be monitored closely. Monitoring should begin within 48 hours of therapy. Vancomycin AUC and trough concentrations should be less than $800 \mu \mathrm{g} \times \mathrm{h} / \mathrm{mL}$ over 24 hours and $15 \mu \mathrm{g} / \mathrm{mL}$, respectively, to minimize acute kidney injury (evidence quality: A-II).

All the recommendations for pediatrics are new for the updated guideline. Pediatric data to support these recommendations are fewer in comparison with adult literature. Given MRSA infections are felt to be similar in adults and children, many pediatric recommendations are extrapolated from adult data and recommendations. The strongest level of evidence in children is the association of acute kidney injury with higher vancomycin exposure, especially with troughs exceeding 15 to $20 \mathrm{mg} / \mathrm{L} .{ }^{6}$ In addition, one pediatric study found an AUC exposure of greater than $800 \mathrm{mg} \times \mathrm{h} / \mathrm{L}$ over 24 hours was strongly associated with risk for acute kidney injury. ${ }^{7}$ These findings suggest that high vancomycin exposure correlates with nephrotoxicity, so with AUC monitoring, the goal exposure should be less than $800 \mathrm{mg} \times \mathrm{hr} / \mathrm{L}$ over 24 hours.

Only one study has evaluated statistical software and prediction of AUC in pediatrics. ${ }^{8}$ A two-concentration approach (peak and trough) outperformed trough-only monitoring for accuracy and precision in determining AUC. While limited to one study, the results are similar to the studies completed in adults, thereby leading to the recommendation of the two-concentration technique in children.

Prospective outcome data are lacking, but multiple retrospective studies have examined $S$ aureus bacteremia in children. Thus far, there have been no studies that have determined the optimal vancomycin exposure required for successful outcomes. 9,10 The proven risks of toxicity are the primary driver for the pediatric guideline change with the outcomes extrapolated from adult data.

\section{CRITIQUE}

\section{Methods in Preparing Guideline}

The main strength of the guideline is that the committee was represented by multiple organizations, which created a multidisciplinary panel of pharmacists and infectious disease physicians with clinical and research expertise in vancomycin dosing. Evidence was graded using an adaptation from the Canadian Task Force on the Periodic Health Examination. ${ }^{11}$ The draft was peer-reviewed by the society organizations and allowed for comments, suggestions, and recommendations.

\section{Sources of Potential Conflict of Interest or Bias}

Disclosures of all authors were reported and identified in the guideline. While many members are involved with pharmaceutical companies through research or speakers' roles, vancomycin, a generic drug, should have minimal conflicts of interest or bias from this involvement.

\section{Generalizability}

Implementation of vancomycin AUC dosing will be hospital dependent due to the implementation-related increase in human 
resources and the cost of clinical software; many hospital systems do not already have the software integrated into their clinical practice. Local guidelines will have to be developed to help clinicians determine which clinical situations require AUC-based dosing vs trough-only monitoring. Pharmacists at many hospitals are primarily responsible for vancomycin monitoring and provide dosing recommendations to physicians. Depending on a hospital system's decision, the workload to determine the optimal vancomycin dose may increase, and it will be important to have close collaboration between hospitalists, pharmacists, and infectious diseases clinicians to appropriately educate clinicians who might be required to dose/monitor vancomycin. One potential way to decrease the burden of monitoring with two concentrations is to use specialized software that can perform complex assessments with only a single concentration. These software applications will still require serious collaboration of the aforementioned practitioners to implement. The variation in guideline adoption will likely be even more significant in pediatrics because the literature is extrapolated and the increased blood draws can be more problematic in pediatric patients.

Furthermore, clinicians should understand the dosing guideline is specifically addressing treatment of MRSA infections and extrapolation to other organisms such as coagulase-negative staphylococcal or methicillin-susceptible $S$ aureus infections should be cautioned. Another caveat to note is that, when the MRSA isolate has an MIC of $2 \mathrm{mg} / \mathrm{L}$ or higher, these infections are associated with poor outcomes when vancomycin is used and alternative agents are recommended.

\section{AREAS IN NEED OF FUTURE STUDY}

Research gaps still remain with appropriate vancomycin drug exposure. In pediatrics, determining the appropriate AUC target will be important given that current recommendations extrapolate from adult data. Future studies can focus on prospective outcome data in both pediatric and adult patients for infections outside of bacteremia or pneumonia, notably central nervous system and osteomyelitis infections. Thresholds for kidney injury will need to be more clearly defined for both adult and pediatric patients. There should also be research emphasis on the appropriate dosing for other non-MRSA invasive infections, notably coagulase-negative staphylococcal infections.

Disclosures: Dr Scheetz reported personal fees for consulting for Achaogen, SIGA technologies, and for serving on an advisory board for Paratek; grants from Merck and Co, Allecra, Nevakar, and SuperTrans Medical; personal fees from Hall, Booth, Smith, PC, and Chambless, Higdon, Richardson, Katz \& Griggs, LLP, for consulting and expert testimony, outside the submitted work.
In addition, Dr. Scheetz has patent US 2019 / 0099500 A1 pending. Dr Murphy reported having received fees from Becton Dickinson for participation to review IDSA guidelines on gastroenteritis. Dr Tang Girdwood has nothing to disclose.

Funding: Dr Murphy and Dr Tang Girdwood are supported by the National Institute of Child Health and Development Cincinnati Pediatric Clinical Pharmacology Postdoctoral Training Program (5T32HD069054-09). Dr Tang Girdwood is also supported by the Cincinnati Children's Hospital Medical Center Arnold W Strauss Fellow Award and Cincinnati Children's Hospital Medical Center Hospital Medicine Fellow Award. Dr Scheetz is supported in part by the National Institute of Allergy and Infectious Diseases award (R21Al149026). The content is solely the responsibility of the authors and does not necessarily represent the official views of the National Institutes of Health.

\section{References}

1. Rybak MJ, Le J, Lodise TP, et al. Therapeutic monitoring of vancomycin for serious methicillin-resistant Staphylococcus aureus infections: a revised consensus guideline and review by the American Society of Health-System Pharmacists, the Infectious Diseases Society of America, the Pediatric Infectious Diseases Society, and the Society of Infectious Diseases Pharmacists. Am J Health Syst Pharm. 2020;77(11):835-864. https://doi.org/10.1093/ajhp/ zxaa036

2. Men P, Li HB, Zhai SD, Zhao RS. Association between the AUC0-24/MIC ratio of vancomycin and its clinical effectiveness: a systematic review and meta-analysis. PLoS One. 2016;11(1):e0146224. https://doi.org/10.1371/journal. pone.0146224

3. Rybak M, Lomaestro B, Rotschafer JC, et al. Therapeutic monitoring of vancomycin in adult patients: a consensus review of the American Society of Health-System Pharmacists, the Infectious Diseases Society of America, and the Society of Infectious Diseases Pharmacists. Am J Health Syst Pharm. 2009;66(1):82-98. https://doi.org/10.2146/ajhp080434

4. Finch NA, Zasowski EJ, Murray KP, et al. A quasi-experiment to study the impact of vancomycin area under the concentration-time curve-guided dosing on vancomycin-associated nephrotoxicity. Antimicrob Agents Chemother. 2017;61(12):e01293-17. https://doi.org/10.1128/aac.01293-17

5. Neely MN, Kato L, Youn G, et al. Prospective trial on the use of trough concentration versus area under the curve to determine therapeutic vancomycin dosing. Antimicrob Agents Chemother. 2018;62(2):e02042-17. https://doi. org/10.1128/aac.02042-17

6. Fiorito TM, Luther MK, Dennehy PH, LaPlante KL, Matson KL. Nephrotoxicity with vancomycin in the pediatric population: a systematic review and meta-analysis. Pediatr Infect Dis J. 2018;37(7):654-661. https://doi.org/10.1097/ inf.0000000000001882

7. Le J, Ny P, Capparelli E, et al. Pharmacodynamic characteristics of nephrotoxicity associated with vancomycin use in children. J Pediatric Infect Dis Soc. 2015;4(4):e109-e116. https://doi.org/10.1093/jpids/piu110

8. Le J, Ngu B, Bradley JS, et al. Vancomycin monitoring in children using bayesian estimation. Ther Drug Monit. 2014;36(4):510-518. https://doi. org/10.1097/ftd.0000000000000039

9. Hahn A, Frenck RW Jr, Allen-Staat M, Zou Y, Vinks AA. Evaluation of target attainment of vancomycin area under the curve in children with methicillin-resistant Staphylococcus aureus bacteremia. Ther Drug Monit. 2015;37(5):619625. https://doi.org/10.1097/ftd.0000000000000190

10. McNeil JC, Kok EY, Forbes AR, et al. Healthcare-associated Staphylococcus aureus bacteremia in children: evidence for reverse vancomycin creep and impact of vancomycin trough values on outcome. Pediatr Infect Dis J. 2016;35(3):263-268. https://doi.org/10.1097/inf.0000000000000991

11. The periodic health examination. Canadian Task Force on the Periodic Health Examination. Can Med Assoc J. 1979;121(9):1193-1254. 